\title{
COMPOSICIÓN Y PATRONES DE DISTRIBUCIÓN GEOGRÁFICA DE LA FLORA DEL BOSQUE DE OYAMEL del Cerro Tláloc, México
}

\author{
Arturo SÁnchez-GonZÁlez ${ }^{1,3}$, Lauro López-Mata² Y Heike Vibrans² \\ ${ }^{1}$ Centro de Investigaciones Biológicas, Universidad Autónoma del Estado de Hidalgo, Ciudad \\ Universitaria, km 4.5 Carretera Pachuca-Tulancingo, Pachuca 42184, Hidalgo, México. \\ 2 Programa de Botánica, Colegio de Postgraduados, km 36.5 Carretera México-Texcoco, Montecillo, \\ Estado de México, C.P. 56230, México. Tel. 58045 947, Fax (595) 9520247. \\ ${ }_{3}^{3}$ Autor para la correspondencia. Tel. (771) 717-2000; correo-e: arturosg@uaeh.edu.mx
}

\begin{abstract}
Resumen: Se analizó la composición y la distribución de las familias, los géneros y las especies de plantas vasculares del bosque de oyamel del Cerro Tláloc en el norte de la Sierra Nevada. En un intervalo altitudinal de 3,000 a 3,500 m se exploró el bosque maduro de oyamel para recolectar el mayor número posible de especímenes durante 16 meses consecutivos. Se encontraron 44 familias, 94 géneros y 137 especies. Tres cuartas partes de las especies pertenecen a sólo 15 familias, destacando Asteraceae, Poaceae, Scrophulariaceae, Geraniaceae, Onagraceae y Pinaceae. La mayoría de las familias y los géneros son de amplia distribución; aproximadamente $88 \%$ de las especies son exclusivas de América, de las cuales $32 \%$ son endémicas de México y $31 \%$ de México-Centroamérica. Solamente 6.6\% de las especies recolectadas son exóticas, lo que sugiere que los cambios en la composición florística del bosque son consecuencia de las actividades humanas.
\end{abstract}

Palabras clave: Abies religiosa, bosque de oyamel, distribución geográfica, florística, Sierra Nevada.

\begin{abstract}
The floristic composition and the distribution of families, genera, and species of vascular plants of the fir forest of Cerro Tláloc, on the northern Sierra Nevada, were analyzed. In an altitudinal range from 3,000 to 3,500 m, the largest possible number of vascular plants was collected during 16 months. There were 44 families, 94 genera, and 137 species. Three fourths of the species belong to only 15 families, among which Asteraceae, Poaceae, Scrophulariaceae, Geraniaceae, Onagraceae, and Pinaceae stand out. Most families and genera have a wide distribution; approximately $88 \%$ of the species are exclusive of the Americas, among which 32\% are endemic to Mexico and 31\% to Mexico-Central America. Only 6.6\% of the species are exotic, suggesting changes in the floristic composition of the fir forest as a consequence of human activities.
\end{abstract}

Key words: Abies religiosa, fir forest, floristics, geographic distribution, Sierra Nevada.

$\mathbf{L}$ os bosques de oyamel (Abies spp.), típicos de la zona ecológica templada subhúmeda de México (Toledo et al., 1993), poseen características florísticas y ecológicas especiales, asociadas con factores climáticos y edáficos. Aproximadamente $82 \%$ de la superficie donde se desarrollan corresponde a zonas montañosas en altitudes entre 2,400 y 3,500 m s.n.m. (Rzedowski, 1978; Sánchez-González y López-Mata, 2005).

Las áreas continuas más extensas de estos bosques se localizan en las serranías que circundan al Valle de México, donde Abies religiosa (Kunth) Cham. et Schltdl. domina el estrato arbóreo superior. El estudio de Madrigal-Sánchez (1967) constituye una referencia importante sobre la com-

posición florística y la estructura de los bosques de oyamel del Valle de México. Desde entonces se ha ahondado poco en su conocimiento ecológico (Ern, 1976; Anaya-Lang et al., 1980; Velázquez y Cleef, 1993; Nieto de Pascual, 1995; Cornejo-Tenorio et al., 2003; Giménez de Azcárate et al., 2003; Sánchez-González y López-Mata, 2003) y florístico (Hernández-Rosales, 1995; Sánchez-González et al., 2005). Los estudios biogeográficos de los bosques de oyamel de México son escasos a nivel de familia y género (Madrigal-Sánchez, 1967; Rzedowski, 1978) y no existen a nivel de especie.

Los bosques de oyamel muestran un patrón de distribución espacial discontinuo análogo a un archipiélago 
(Vázquez-García, 1993; Rzedowski, 1998). Este hecho presenta una situación ideal para estudiar la distribución de sus especies, ya que la composición de los bosques templados de México varía considerablemente entre regiones e inclusive de un sitio a otro dentro de cada región (Challenger, 1998).

La Faja Volcánica Transmexicana (sensu FerrusquíaVillafranca, 1998) es una región montañosa de especial importancia, al constituir una zona de transición natural entre los reinos biogeográficos neártico y neotropical (Morrone, 2004) y una zona de conexión de las biotas de la Sierra Madre Oriental y la Sierra Madre Occidental.

Las perturbaciones ocasionadas por el vulcanismo característico de la Faja Volcánica Transmexicana en el pasado geológico generaron una enorme heterogeneidad de hábitats que probablemente facilitó la especiación y la hibridación de especies (Challenger, 1998). La coexistencia de especies de diferente origen contribuye a que esta amplia región de México sea una de las más ricas del mundo (Graham, 1998; Turner y Nesom, 1998; Morrone, 2004), pero también una de las más habitadas e intensamente modificadas por el hombre desde hace miles de años (Calderón de Rzedowski y Rzedowski, 2001).

Flores-Mata et al. (1971) estimaron que los bosques de oyamel ocupaban $0.16 \%$ de la superficie de México; sin embargo, este porcentaje está disminuyendo drásticamente debido principalmente a los desmontes, la tala inmoderada, los incendios, el pastoreo, la contaminación ambiental y las plagas y enfermedades (Rzedowski, 1978; Challenger, 1998; Alvarado-Rosales y Hernández-Tejeda, 2002; Sánchez-González et al., 2005).

Dada la importancia biológica, económica y la presión a que está sometida la zona ecológica templada subhúmeda de México y en particular el bosque de Abies, es urgente conocer estos sistemas desde un punto de vista ecológico, florístico y biogeográfico. Además de los beneficios económicos, biológicos y espirituales, los bosques de oyamel destacan por sus funciones de protección contra la erosión, captura de carbono, captación de agua y regulación de la escorrentía (Hernández, 1985), lo que apoya la necesidad de evaluar su estado natural actual.

Los objetivos de esta investigación fueron describir la composición florística y la distribución geográfica actual de las familias, los géneros y las especies del bosque de oyamel del Cerro Tláloc, en el norte de la Sierra Nevada.

\section{Materiales y métodos}

El área de estudio se localiza en la provincia morfotectónica conocida como Faja Volcánica Transmexicana, en la región norte de la Sierra Nevada, en el oriente del estado de México y se extiende sobre el declive oeste del Cerro Tláloc, entre $19^{\circ} 23^{\prime} 43^{\prime \prime}$ y $19^{\circ} 28^{\prime} 37^{\prime \prime}$ de latitud norte y entre $98^{\circ} 42^{\prime} 51^{\prime \prime}$ y $98^{\circ} 48^{\prime} 12^{\prime \prime}$ de longitud oeste (figura 1 ). Las elevaciones más notables corresponden a los cerros Tláloc (4,120 m), Telapón (4,060 m), Yoloxóchitl (3,900 m) y Los Potreros (3,900 m; Sánchez-González, 2004). El clima es templado húmedo, con régimen de lluvias en verano, con una oscilación de la temperatura promedio mensual entre 5 y $7^{\circ} \mathrm{C}$. En la zona de lomeríos la precipitación anual varía entre 800 y $1,200 \mathrm{~mm}$ y la temperatura media anual entre 10 y $18^{\circ} \mathrm{C}$ (Ortiz-Solorio y Cuanalo de la Cerda, 1977). Los suelos son negros, profundos, muy ricos en materia orgánica y de textura media. A lo largo del declive oeste del Cerro Tláloc se presentan seis tipos de vegetación natural dominante: encinar arbustivo, bosque de encino, bosque mixto, bosque de oyamel, bosque de pino y zacatonal alpino. El bosque maduro de oyamel crece en cañadas y partes bajas de las laderas, en sitios húmedos y protegidos con pendientes mayores de $40 \%$. La precipitación anual fluctúa de 900 a $1,000 \mathrm{~mm}$ y la temperatura media anual de 10 a $12^{\circ} \mathrm{C}$. Los suelos son de textura media (migajones o francos), profundos, oscuros y ricos en materia orgánica; los valores de $\mathrm{pH}$ varían de 5.5 a 7.1 (Sánchez-González y López-Mata, 2003).

El estudio se realizó en el declive oeste del norte de la Sierra Nevada, en un intervalo altitudinal de 3,000 a 3,500 $\mathrm{m}$ donde se recorrieron los bosques maduros de oyamel reportados por Sánchez-González y López-Mata (2003), con el fin de recolectar el mayor número posible de especímenes. El trabajo de campo se llevó a cabo durante 16 meses consecutivos. Los ejemplares recolectados fueron determinados con base en las claves de la Flora del Valle de México (Calderón de Rzedowski y Rzedowski, 2001) y en algunos grupos se recurrió a taxónomos especialistas. Los ejemplares recolectados se depositaron en los herbarios de la División de Ciencias Forestales de la Universidad Autónoma Chapingo (CHAP) y de la Facultad de Estudios Superiores Iztacala (IZTA). La nomenclatura utilizada para los nombres de las especies se basó en Calderón de Rzedowski y Rzedowski (2001) y para las pteridofitas se basó en Mickel y Smith (2004); la forma de vida de las especies se estableció en campo con base en el criterio de Moreno (1984). Los nombres de los autores se abreviaron de acuerdo con Brummitt y Powell (1992). La información de la distribución geográfica actual de las familias y géneros de plantas vasculares se basó en los trabajos de Mabberley (1993), Qian (1999) y Calderón de Rzedowski y Rzedowski (2001), y la distribución de las especies principalmente en el trabajo de Calderón de Rzedowski y Rzedowski (2001).

\section{Resultados}

Composición florística. En los bosques maduros de oyamel analizados se encontraron 44 familias, 94 géneros y 137 especies de plantas vasculares (apéndice 1). El grupo más numeroso de especies correspondió a Magnoliophyta, 


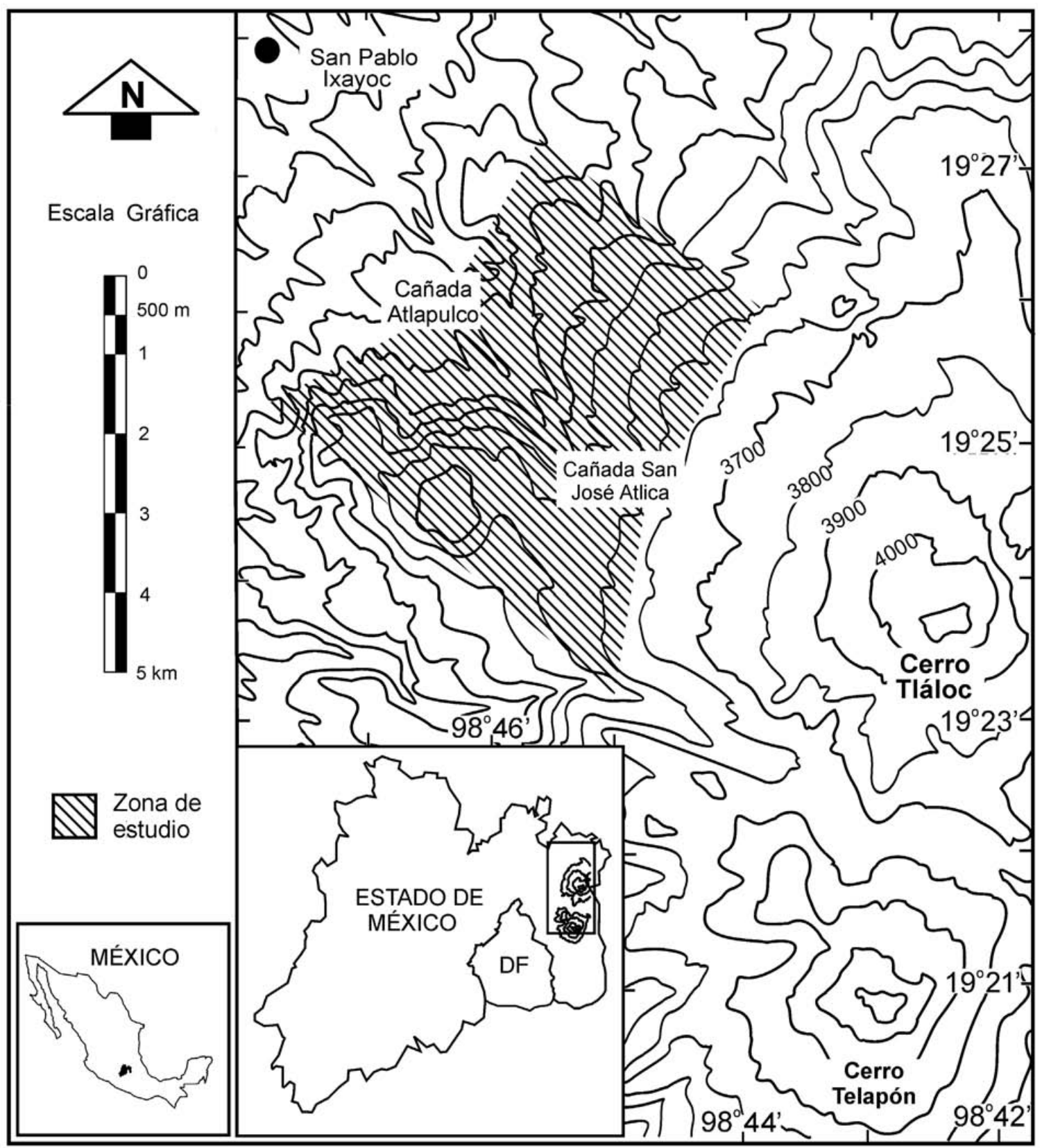

Figura 1. Localización de la zona de estudio.

seguido por Polypodiophyta y por Pinophyta. La clase Magnoliopsida representó más de $78 \%$ y la Liliopsida más de $6 \%$ de las familias, géneros y especies (cuadro 1).

Tres cuartas partes de las especies pertenecen a sólo 15 familias, de las cuales Asteraceae, Poaceae, Scrophulariaceae, Geraniaceae, Onagraceae, Pinaceae, Lamiaceae y Rosaceae son las de mayor riqueza en géneros y especies. La familia Asteraceae es particularmente diversa pues representa $21 \%$ y $27 \%$ de los géneros y especies, respectivamente (cuadro 2 ).

La forma de crecimiento más numerosa fue la herbácea, con un total de 104 especies (76\%); 22 (16\%) especies fueron arbustos y 11 (8\%) árboles. La especie Polypodium californicum Kaulf. se reportó por primera vez para el estado de México durante el desarrollo del presente estudio (Tejero-Díez y Arreguín-Sánchez, 2004). Por otra parte, las especies Corallorrhiza macrantha Schltr., Comarostaphylis discolor (Hook.) Diggs y Cupressus lusitanica Mill. están 
Cuadro 1. Riqueza de los grupos de plantas vasculares del bosque de oyamel del Cerro Tláloc.

\begin{tabular}{lccc}
\hline Grupo & Familias & Géneros & Especies \\
\hline Polypodiophyta & $4(9.1 \%)$ & $5(5.3 \%)$ & $7(5.1 \%)$ \\
Pinophyta & $2(4.5 \%)$ & $3(3.2 \%)$ & $6(4.3 \%)$ \\
Magnoliophyta & & & \\
$\quad$ Liliopsida & $3(6.8 \%)$ & $10(11.7 \%)$ & $17(12.4 \%)$ \\
$\quad$ Magnoliopsida & $35(79.6 \%)$ & $76(79.8 \%)$ & $107(78.2 \%)$ \\
\hline
\end{tabular}

sujetas a protección especial (SEMARNAT, 2002). En un estudio previo, Sánchez-González y López-Mata (2003) encontraron que el estrato arbóreo del bosque de oyamel en el Cerro Tláloc está dominado por Abies religiosa y en menor medida por Alnus acuminata, Berberis moranensis, Pinus hartwegii, P. montezumae y Salix paradoxa. Por su parte, el estrato arbustivo está dominado por Acaena elongata, Buddleia parviflora, Comarostaphylis discolor, Fuchsia microphylla, F. thymifolia, Ribes ciliatum, Salvia fulgens, S. gesnerifolia, Senecio barba-johannis, S. angulifolius, S. cinerarioides y Symphoricarpos microphyllus, y el estrato herbáceo por Alchemilla procumbens, Archibaccharis hieracioides, Brachypodium mexicanum, Didymaea alsinoides, Erigeron galeottii, Eupatorium pazcuarense, Festuca amplissima, Galium aschenbornii, Senecio callosus, S. platanifolius, S. toluccanus y Stevia monardifolia.

\section{Distribución geográfica}

Familias. Las 44 familias de plantas vasculares encontradas en el bosque de oyamel del Cerro Tláloc se asignaron a tres categorías de acuerdo con su distribución actual: 35 (79.5\%) son de amplia distribución, cinco (11.4\%) son tropicales y subtropicales, y cuatro $(9.1 \%)$ del Hemisferio Norte (figura 2). Estas categorías generales se dividieron de acuerdo con la distribución principal de sus elementos. En la categoría de amplia distribución son más abundantes las familias cosmopolitas y subcosmopolitas, con 15 que representan 34\% del total general; las familias que se distribuyen en especial en el Hemisferio Norte fueron nueve (20.4\%), las que se distribuyen principalmente en América fueron cinco $(11.3 \%)$. En las regiones templado-cálidas tres (6.8\%), las de distribución tropical y subtropical fueron dos $(4.5 \%)$ y una familia $(2.3 \%)$ en especial en las regiones templado-frías. En la categoría de familias del Hemisferio Norte, dos familias (4.5\%) son de amplia distribución y dos

Cuadro 2. Número y porcentaje de géneros (G) y especies (E) por familia.

\begin{tabular}{|c|c|c|c|c|c|c|c|c|c|}
\hline Familia & G & E & $\% \mathrm{G}$ & $\% \mathrm{E}$ & Familia & G & E & $\% \mathrm{G}$ & $\% \mathrm{E}$ \\
\hline Asteraceae & 20 & 38 & 21.28 & 27.60 & Athyriaceae & 1 & 1 & 1.06 & 0.74 \\
\hline Poaceae & 8 & 13 & 8.51 & 9.42 & Berberidaceae & 1 & 1 & 1.06 & 0.74 \\
\hline Scrophulariaceae & 5 & 7 & 5.32 & 5.10 & Betulaceae & 1 & 1 & 1.06 & 0.74 \\
\hline Geraniaceae & 2 & 5 & 2.13 & 3.62 & Boraginaceae & 1 & 1 & 1.06 & 0.74 \\
\hline Onagraceae & 4 & 5 & 4.26 & 3.62 & Commelinaceae & 1 & 1 & 1.06 & 0.74 \\
\hline Pinaceae & 2 & 5 & 2.13 & 3.62 & Cruciferae & 1 & 1 & 1.06 & 0.74 \\
\hline Lamiaceae & 3 & 4 & 3.19 & 2.90 & Cupressaceae & 1 & 1 & 1.06 & 0.74 \\
\hline Rosaceae & 4 & 4 & 4.26 & 2.90 & Dryopteridaceae & 1 & 1 & 1.06 & 0.74 \\
\hline Aspleniaceae & 1 & 3 & 1.06 & 2.18 & Euphorbiaceae & 1 & 1 & 1.06 & 0.74 \\
\hline Caryophyllaceae & 2 & 3 & 2.14 & 2.18 & Fagaceae & 1 & 1 & 1.06 & 0.74 \\
\hline Ericaceae & 3 & 3 & 3.19 & 2.18 & Grossulariaceae & 1 & 1 & 1.06 & 0.74 \\
\hline Gentianaceae & 3 & 3 & 3.19 & 2.18 & Hydrophyllaceae & 1 & 1 & 1.06 & 0.74 \\
\hline Orchidaceae & 2 & 3 & 2.14 & 2.18 & Loganiaceae & 1 & 1 & 1.06 & 0.74 \\
\hline Pyrolaceae & 2 & 3 & 2.14 & 2.18 & Plantaginaceae & 1 & 1 & 1.06 & 0.74 \\
\hline Caprifoliaceae & 2 & 2 & 2.14 & 1.45 & Polemoniaceae & 1 & 1 & 1.06 & 0.74 \\
\hline Rubiaceae & 2 & 2 & 2.14 & 1.45 & Polygonaceae & 1 & 1 & 1.06 & 0.74 \\
\hline Polypodiaceae & 2 & 2 & 2.14 & 1.45 & Portulacaceae & 1 & 1 & 1.06 & 0.74 \\
\hline Fabaceae & 1 & 2 & 1.06 & 1.45 & Resedaceae & 1 & 1 & 1.06 & 0.74 \\
\hline Loranthaceae & 1 & 2 & 1.06 & 1.45 & Salicaceae & 1 & 1 & 1.06 & 0.74 \\
\hline Oxalidaceae & 1 & 2 & 1.06 & 1.45 & Saxifragaceae & 1 & 1 & 1.06 & 0.74 \\
\hline Solanaceae & 1 & 2 & 1.06 & 1.45 & Urticaceae & 1 & 1 & 1.06 & 0.74 \\
\hline Umbelliferae & 1 & 2 & 1.06 & 1.45 & Valerianaceae & 1 & 1 & 1.06 & 0.74 \\
\hline
\end{tabular}


(4.5\%) se distribuyen en especial en Europa y Asia. En la categoría de familias tropicales y subtropicales, tres familias son de distribución tropical $(6.8 \%)$ y dos son de amplia distribución (4.5\%).

Géneros. Para indicar la distribución geográfica actual de los 94 géneros se utilizaron las mismas categorías generales que para las familias: $50(53.2 \%)$ géneros son de amplia distribución, 24 (25.6\%) se distribuyen en el Hemisferio Norte, 17 (18\%) son exclusivos del Continente Americano $\mathrm{y}$ tres $(3.2 \%)$ se distribuyen en las regiones tropicales y subtropicales (figura 2).

Cada una de estas categorías generales se dividió, a su vez, de acuerdo con la distribución de sus elementos (figura 2). En la categoría de amplia distribución, 14 (14.9\%) géneros son cosmopolitas y sub-cosmopolitas, uno (1.1\%) se encuentra en especial en el Hemisferio Norte, cuatro (4.2\%) en el Hemisferio Sur, uno (1.1\%) es afín a zonas tropicales y subtropicales, seis $(6.4 \%)$ en zonas templadocálidas, 14 (14.9\%) en especial en zonas templado-frías y $10(10.6 \%)$ en América. En la categoría de géneros del Hemisferio Norte seis $(6.3 \%)$ son de amplia distribución, $13(13.7 \%)$ de las zonas templadas y frías, una (2\%) se distribuye en especial en Eurasia y el Mediterráneo y cuatro (4.2\%) en el continente americano.

La categoría de géneros de zonas tropicales y subtropicales comprende dos $(2.1 \%)$ de distribución americana y uno $(1.0 \%)$ pantropical. De los géneros exclusivos de América, cinco $(5.3 \%)$ se distribuyen de Norte a Sudamérica y tres $(3.2 \%)$ de México a Sudamérica, dos (2.1\%) de Estados Unidos a Centroamérica, cuatro (4.2\%) de México a Centroamérica y tres $(3.2 \%)$ se encuentran en especial en Norteamérica.

Especies. La distribución geográfica de las 137 especies recolectadas se clasificó en 10 categorías (cuadro 3; figura 2). Un gran porcentaje (87.7\%) de las especies son exclusivas de América: $32 \%$ de las especies son endémicas de
México, 31\% son exclusivas de México-Centroamérica, $10 \%$ son exclusivas de México-Sudamérica y $12.3 \%$ se encuentran distribuidas en otras regiones del mundo. Además, se encontraron a las especies exóticas (sensu Villaseñor y Espinosa-García, 2004) Erodium cicutarium, Vulpia myuros, Polygonum aviculare, Reseda luteola, Veronica persica, Veronica serpyllifolia, Poa annua, Sonchus oleraceus y Taraxacum officinale formando parte de la vegetación natural $(6.6 \%)$.

En el cuadro 3 se muestra que la mayoría de las 137 especies se distribuye en México, México-Centroamérica, México-Sudamérica y Norte-Centroamérica. Entre las formas de crecimiento arbóreo y arbustivo todas las especies son exclusivas de América, mientras que 12.3\% de las especies herbáceas se distribuyen también en otras regiones del planeta.

\section{Discusión}

Los bosques templados de la Faja Volcánica Transmexicana poseen una composición florística en la que un número reducido de familias contienen a la mayoría de las especies en cada localidad (Vega-Aviña, 1982; Boyas-Delgado, 1993; Hernández-Rosales, 1995; Orozco-Villa, 1995; Silva-Pérez, 1998; Sánchez-González et al., 2005). En el mismo escenario, 14 de las 44 familias del bosque de oyamel del Cerro Tláloc contienen $72 \%$ de las especies recolectadas en el área. Estas cifras corroboran el patrón descrito por Tolmatschew (1971) para otras regiones templadas del mundo: aproximadamente 10 familias contienen a la mayoría de las especies de una flora, y el porcentaje de contribución de estas familias al número total de especies es relativamente constante para floras distintas pero de la misma región florística. En contraste, en floras de distintas regiones florísticas el porcentaje de contribución de las mismas familias es muy variable.

Cuadro 3. Distribución geográfica de las especies del bosque de oyamel por forma de crecimiento.

\begin{tabular}{lcccc}
\hline \multirow{2}{*}{ Distribución } & & & Forma de crecimiento (\%) \\
\cline { 3 - 4 } & Total & árboles & arbustos & hierbas \\
\hline Amplia distribución & & 0 & 0 & 5.0 \\
Hemisferio Norte & 5.0 & 0 & 0 & 6.5 \\
América-África & 6.5 & 0 & 0 & 0.8 \\
Canadá o Estados Unidos-México & 0.8 & 0 & 0.7 & 2.9 \\
Norte-Centroamérica & 3.6 & 0.7 & 1.5 & 4.3 \\
Norte-Sudamérica & 6.5 & 0 & 0 & 2.9 \\
México & 2.9 & 2.9 & 8.7 & 21.0 \\
México-Centroamérica & 32.6 & 3.6 & 3.6 & 24.0 \\
México-Sudamérica & 31.2 & 0.7 & 0 & 8.0 \\
Centroamérica-Sudamérica, Las Antillas & 10.1 & 0 & 0.4 \\
\hline
\end{tabular}



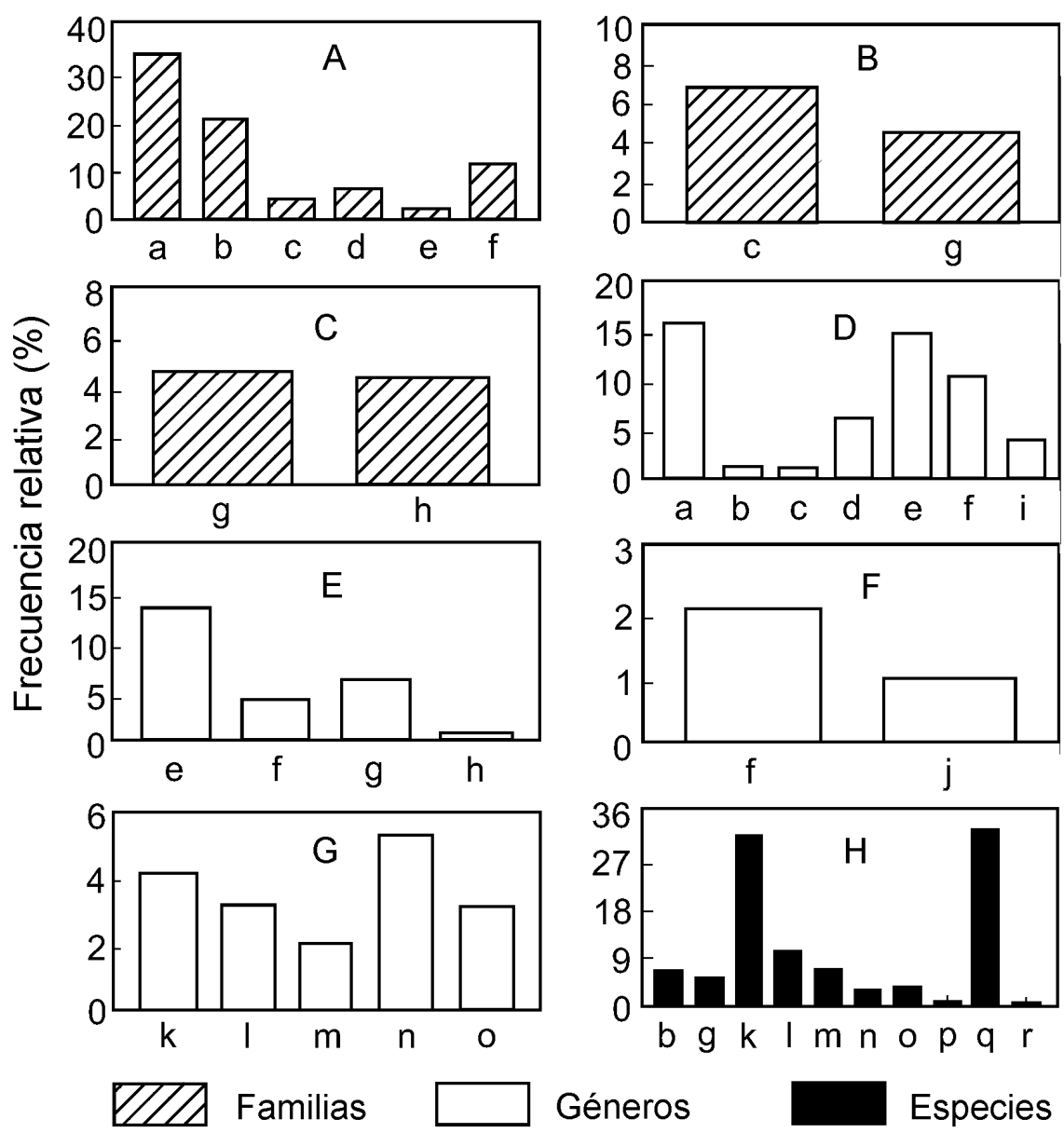

Figura 2. Distribución geográfica de las familias, géneros y especies. A: Familias de amplia distribución, B: Familias tropicales y subtropicales, C: Familias del Hemisferio Norte, D: Géneros de amplia distribución, E: Géneros del Hemisferio Norte, F: Géneros tropicales y subtropicales, G: Géneros de América, H: Distribución actual de las especies. a: Cosmopolitas y sub-cosmopolitas, b: Hemisferio Norte, c: preferentemente tropicales y subtropicales, d: preferentemente de zonas templado-cálidas, e: preferentemente de zonas templado-frías, f: preferentemente en América, g: de amplia distribución, h: euroasiáticas, i: Hemisferio Sur, j: pantropicales, k: México-Centroamérica, 1: México-Sudamérica, m: Norteamérica-Centroamérica, n: Norteamérica-Sudamérica, o: Norteamérica, p: América-África, q: México, r: Centroamérica-Sudamérica-Las Antillas.

Rzedowski (1998) opina que la flora de las regiones templadas subhúmedas de México se ha conformado en gran proporción a base de elementos que existen también en otras partes del mundo, pero un considerable número de tales elementos experimentó una extensa radiación secundaria a nivel local que originó la alta diversidad actual. En concordancia con esta afirmación, la mayoría de las familias de plantas vasculares registradas en el bosque de oyamel del Cerro Tláloc se localizan también en otras regiones del mundo.

De acuerdo con Tolmatschew (1971), la contribución de las familias más ricas al número total de especies de una flora determinada puede ser alta si hubo catástrofes en el pasado geológico. México tiene una historia geológica compleja responsable de la abrupta topografía y del amplio mosaico de climas y tipos de vegetación. En el caso específico de la Faja Volcánica Transmexicana, el vulcanismo característico de esta región en el pasado geológico generó una enorme variedad de hábitats, lo que permitió la especiación y la hibridación (Challenger, 1998).

En la Faja Volcánica Transmexicana las familias Asteraceae, Lamiaceae, Fabaceae, Pinaceae, Poaceae y Rubiaceae han alcanzado una alta diversificación biológica (Graham, 1998; Rzedowski, 1998; Turner y Nesom, 1998). La familia Asteraceae es la más diversa en las regiones templadas de México, donde representa entre 15 y $20 \%$ de la flora genérica (Rzedowski, 1978; Challenger, 1998). En el bosque de oyamel analizado la familia Asteraceae contiene 
$21.3 \%$ de los géneros y $27.5 \%$ de las especies, lo que la hace también la mejor representada.

A nivel de género, se considera que la flora de la zona templada subhúmeda de México está compuesta por elementos de afinidad neotropical y holártica, en particular andina y del oeste de Norteamérica, respectivamente, así como de un número menor de taxa endémicos (Rzedowski, 1978). La distribución actual de cada uno de los géneros del bosque de oyamel analizado es consecuencia de sus diferentes tiempos y espacios de origen, mecanismos de dispersión y de sus propias adaptaciones paleoecológicas (Rzedowski, 1991; Qian, 1999): la mayoría son de amplia distribución (53.2\%), del hemisferio norte (25.6\%) o exclusivos o de distribución actual en América (20\%).

La flora de las regiones montañosas de México, cubiertas por bosques de coníferas y de encino, es casi tan rica en endemismos como la de las zonas secas debido a un largo e intenso periodo de evolución in situ, resultando en un centro de diversificación secundario para muchos taxa (De Luna, 1985; Rzedowski, 1991). Rzedowski (1998) menciona que géneros como Eupatorium, Pinus, Senecio y Stevia han tenido un importante centro de diversificación en las regiones montañosas de México. Otros estudios realizados en diferentes localidades de la Faja Volcánica Transmexicana (Vega-Aviña, 1982; Boyas-Delgado, 1993; Hernández-Rosales, 1995; Miranda-Jiménez y GonzálezOrtiz, 1993; Cornejo-Tenorio et al., 2003; SánchezGonzález et al., 2005) citan como más diversos, además de los géneros anteriores, a Asplenium, Baccharis, Geranium, Gnaphalium y Solanum (todos ellos con tres o más especies en el bosque de oyamel estudiado).

Rzedowski (1998) denominó "Megaméxico 3" al área que comprende el sur de Estados Unidos, todo México y el norte de América Central, hasta la depresión de Nicaragua, y sugirió considerarla una unidad fitogeográfica natural con un alto porcentaje de endemismos. Halffter (2003) definió a la "zona de transición mexicana" como el área donde se superponen elementos neárticos y neotropicales provenientes del suroeste de los Estados Unidos, México y la mayor parte de América Central. Esta zona de transición es de especial importancia evolutiva, pues los límites entre regiones biogeográficas son áreas de intensa interacción biótica promovida por cambios ecológicos e históricos (Morrone, 2004, 2006). Si se consideran los criterios de Rzedowski (1998) y Halffter (2003), en el bosque de oyamel del Cerro Tláloc predominan a nivel de especie los elementos endémicos, ya que más de $76 \%$ de las especies son exclusivas de Megaméxico 3 (que corresponde aproximadamente a la zona de transición mexicana) y $32.6 \%$ son endémicas de México. Muchas de estas especies debieron originarse en el pasado geológico reciente por los cambios climáticos ocurridos durante el Pleistoceno en las principales cadenas montañosas de México (Graham, 1998; Rzedowski, 1998).
Las especies presentes en el bosque de oyamel del Cerro Tláloc tienen una relación más estrecha con Centroamérica $(31.2 \%)$ que con el resto del continente americano. Esta fuerte afinidad florística entre México y Centroamérica ha sido ampliamente documentada (Vega-Aviña, 1982; Almeida-Leñero, 1997; Islebe y Velázquez, 1994; Rzedowski, 1998; Vibrans, 1998; Sánchez-González, 2004).

La formación del Istmo de Panamá en el Plioceno creó un puente entre Norte y Sudamérica que posibilitó la migración de la flora y la fauna, fenómeno conocido como "el gran intercambio americano" (Marshall et al., 1979). Las fases de calentamiento y enfriamiento mundial también influyeron en los patrones de migración y de diversificación. En este escenario dinámico, es probable que las oportunidades para la especiación fueran más elevadas que las actuales para las plantas (Graham, 1998; Morley, 2003).

El estudio realizado por Sánchez-González y LópezMata (2003) confirma que el bosque de oyamel del Cerro Tláloc conserva la estructura típica de otros bosques localizados en la Faja Volcánica Transmexicana: unas pocas especies dominantes y un gran número de especies raras (Madrigal-Sánchez, 1967; Nieto de Pascual, 1995; Sánchez-González et al., 2005), de las cuales $6.6 \%$ se consideran exóticas (Villaseñor y Espinosa-García, 2004). La alta riqueza de especies arbustivas y herbáceas sugiere que este tipo de vegetación está sujeto a perturbaciones humanas recurrentes. En el bosque de oyamel analizado son comunes el pastoreo de ganado bovino y ovino, los incendios forestales y la extracción clandestina de madera debido a que se localiza en las inmediaciones de uno de los complejos urbanos más grandes del mundo (MadrigalSánchez, 1967; Rzedowski, 1978; Alvarado-Rosales y Hernández-Tejeda, 2002; Sánchez-González y LópezMata, 2003; Sánchez-González et al., 2005).

Los resultados de este estudio aportan nueva información sobre la distribución actual de las especies y sobre la composición florística de los bosques maduros de oyamel del Valle de México: una de las especies recolectadas durante el trabajo de campo resultó ser nuevo reporte (Tejero-Díez y Arreguín-Sánchez, 2004), lo que demuestra que el conocimiento de este tipo de vegetación es aún incompleto.

\section{Agradecimientos}

El primer autor agradece al Consejo Nacional de Ciencia y Tecnología la beca otorgada para realizar sus estudios doctorales. Agradecemos a dos revisores anónimos, así como a Joaquín Giménez de Azcárate y a Jorge A. Meave por sus comentarios y sugerencias que mejoraron enormemente el manuscrito original. 


\section{Literatura citada}

Almeida-Leñero L. 1997. Vegetación, fitogeografía y paleoecología del zacatonal alpino y bosques montanos de la región central de México. Tesis doctoral, Hugo de Vries Laboratory, Departament of Palynology and Paleo/Actuo-Ecology, University of Amsterdam, Amsterdam, $256 \mathrm{pp}$.

Alvarado-Rosales D. y Hernández-Tejeda T. 2002. Decline of sacred fir in the Desierto de los Leones National Park. En: Fenn M.E., Bauer L.I. y Hernández-Tejeda T. Eds. Urban Air Pollution and Forests: Resources at Risk in the Mexico City Air Basin, pp. 243-260, Springer-Verlag, Nueva York.

Anaya-Lang M.L., Hernández-Sánchez R. y Madrigal-Sánchez X. 1980. La Vegetación y los Suelos de un Transecto Altitudinal del Declive Occidental del Iztaccíhuatl (México). Boletín Técnico 65, Instituto Nacional de Investigaciones Forestales, México, D.F.

Boyas-Delgado J.C. 1993. Flora Fanerogámica del Campo Experimental "San Juan Tetla", Puebla. 2a ed. Folleto Técnico No. 114, Secretaría de Agricultura y Recursos Hidráulicos, Instituto de Investigaciones Forestales y Agropecuarias, División Forestal, México, D.F.

Brummitt R.K. y Powell C.E. 1992. Authors of Plant Names. Royal Botanical Gardens, Kew.

Calderón de R. G. y Rzedowski J. Eds. 2001. Flora Fanerogámica del Valle de México. 2a ed., Instituto de Ecología, A.C. y Comisión Nacional para el Conocimiento y Uso de la Biodiversidad, Pátzcuaro.

Challenger A. 1998. Utilización y Conservación de los Ecosistemas Terrestres de México. Pasado, Presente y Futuro. Comisión Nacional para el Conocimiento y Uso de la Biodiversidad, Instituto de Biología, Universidad Nacional Autónoma de México y Agrupación Sierra Madre, S.C., México, D.F.

Cornejo-Tenorio G., Casas A., Farfán B., Villaseñor J.L. e IbarraManríquez G. 2003. Flora y vegetación de las zonas núcleo de la reserva de la biosfera Mariposa Monarca, México. Boletín de la Sociedad Botánica de México 73:43-62.

De Luna E. 1985. Afinidades fitogeográficas de los musgos de los extremos del Eje Neovolcanico, México. Biotica 10:235-255.

Ern H. 1976. Descripción de la Vegetación Montañosa de los Estados Mexicanos de Puebla Tlaxcala. Willdenowia 10:1128.

Ferrusquía-Villafranca I. 1998. Geología de México: una sinopsis. En: Ramamoorthy T.P., Bye R., Lot A. y Fa. J. Comp. Diversidad Biológica de México: Orígenes y Distribución, pp. 3-108, Instituto de Biología, Universidad Nacional Autónoma de México, México, D.F.

Flores-Mata G., Jiménez-López J., Madrigal-Sánchez X., Moncayo-Ruiz F. y Takaki-Takaki F. 1971. Memoria del Mapa de Tipos de Vegetación de la Republica Mexicana. Secretaría de Recursos Hidráulicos, México, D.F.

Giménez de Azcárate J., Ramírez M.I. y Pinto M. 2003. Las comunidades vegetales de la Sierra de Angangueo (estados de Michoacán y México, México): clasificación, composición y distribución. Lazaroa 24:87-111.

Graham A. 1998. Factores históricos de la diversidad biológica de México. En: Ramamoorthy T.P., Bye R., Lot A. y Fa. J. Comps. Diversidad Biológica de México: Orígenes y Distribución, pp. 109-127, Instituto de Biología, Universidad Nacional
Autónoma de México, México, D.F.

Halffter G. 2003. Biogeografía de la entomofauna de montaña de México y América Central. En: Morrone J.J. y Llorente J. Eds. Una Perspectiva Latinoamericana de la Biogeografía, pp. 8797, Las Prensas de Ciencias, Facultad de Ciencias, Universidad Nacional Autónoma de México, México, D.F.

Hernández M.E. 1985. Distribución y utilidad de los Abies en México. Boletín del Instituto de Geografía 15:75-118.

Hernández-Rosales M.R.A. 1995. Estudio florístico-fanerogámico del Parque Nacional El Chico, Estado de Hidalgo. Tesis de Licenciatura (Biología), Escuela Nacional de Estudios Profesionales Iztacala, Universidad Nacional Autónoma de México, Los Reyes Iztacala, Estado de México, 73 pp.

Islebe G.A. y Velázquez A. 1994. Affinity among mountain ranges in Megamexico: A phytogeographical scenario. Vegetatio 115:1-9.

Mabberley D.J. 1993. The Plant Book. A Portable Dictionary of the Higher Plants. 2a ed., Cambridge University Press, Cambridge.

Madrigal-Sánchez X. 1967. Contribución al Conocimiento de la Ecología de los Bosques de Oyamel (Abies religiosa (H.B.K.) Schl. \& Cham.) en el Valle de México. Boletín Técnico No. 18, Instituto Nacional de Investigaciones Forestales, México, D.F.

Marshall L.G., Butler R.F., Drake R.E., Curtis G.H. y Tedford R.H. 1979. Calibration of the Great American Interchange. Science 204:272-279.

Mickel J.T. y Smith A.R. 2004. The Pteridophytes of Mexico. Memoirs of the New York Botanical Garden, New York Botanical Garden Press, Nueva York.

Miranda-Jiménez M.E. y González-Ortiz M.A. 1993. Estudio de la vegetación y florística de la mesa basáltica de Holotepec, distrito de Tenango del Valle, Estado de México. Tesis de Licenciatura (Biología), Escuela Nacional de Estudios Profesionales Iztacala, Universidad Nacional Autónoma de México, Los Reyes Iztacala, Estado de México, 74 pp.

Moreno N.P. 1984. Glosario Botánico Ilustrado. Compañía Editorial Continental, S.A., México, D.F.

Morley R.J. 2003. Interplate dispersal paths for megathermal angiosperms. Perspectives in Plant Ecology, Evolution and Systematics 6:5-20.

Morrone J.J. 2004. Panbiogeografia, componentes bióticos y zonas de transición. Revista Brasileira de Entomologia 48:149162 .

Morrone J.J. 2006. Biogeographic areas and transition zones of Latin America and the Caribbean islands based on panbiogeographic and cladistic analysis of the entomofauna. Annual Review of Entomology 51:467-494.

Nieto de Pascual P.C. 1995. Estudio sinecológico del bosque de oyamel de la cañada de Contreras, Distrito Federal. Revista Ciencia Forestal en México 20:3-34.

Orozco-Villa M. 1995. Vegetación del municipio de Temascaltepec, Estado de México. Tesis de Licenciatura (Biología), Escuela Nacional de Estudios Profesionales Iztacala, Universidad Nacional Autónoma de México, Los Reyes Iztacala, Estado de México, 89 pp.

Ortiz-Solorio C. y Cuanalo de la Cerda H. 1977. Levantamiento Fisiográfico del Área de Influencia de Chapingo. Colegio de Postgraduados, Escuela Nacional de Agricultura, Chapingo, Estado de México.

Qian H. 1999. Floristic analysis of vascular plant genera of North 
America north of Mexico: Characteristics of phytogeography. Journal of Biogeography 26:1307-1321.

Rzedowski J. 1978. Vegetación de México. Limusa, México, D.F.

Rzedowski J. 1991. El endemismo en la flora fanerogámica mexicana: una apreciación analítica preliminar. Acta Botanica Mexicana 15:47-64.

Rzedowski J. 1998. Diversidad y orígenes de la flora fanerogámica de México. En: Ramamoorthy T.P., Bye R., Lot A. y Fa J. Comp. Diversidad Biológica de México: Orígenes y Distribución, pp. 129-145, Instituto de Biología, Universidad Nacional Autónoma de México, México, D.F.

Sánchez-González A. 2004. Análisis sinecológico, florístico y biogeográfico de la vegetación del norte de la Sierra Nevada. Tesis doctoral, Especialidad en Botánica, Colegio de Postgraduados, Montecillo, Estado de México, 153 pp.

Sánchez-González A. y López-Mata L. 2003. Clasificación y ordenación de la vegetación del norte de la Sierra Nevada, a lo largo de un gradiente altitudinal. Anales del Instituto de Biología, Serie Botánica 74:47-71.

Sánchez-González A. y López-Mata L. 2005. Plant species richness and diversity along an altitudinal gradient in the Sierra Nevada, Mexico. Diversity and Distributions 11:567-575.

Sánchez-González A., López-Mata L. y Granados-Sánchez D. 2005. Semejanza florística entre los bosques de Abies religiosa (H.B.K.) Cham. \& Schltdl. de la Faja Volcánica Transmexicana. Boletín del Instituto de Geografía 56:62-76.

SEMARNAT [Secretaría del Medio Ambiente y Recursos Naturales]. 2002. Norma Oficial Mexicana NOM-059-ECOL2001, Protección ambiental-Especies nativas de México de flora y fauna silvestres-Categorías de riesgo y especificaciones para su inclusión, exclusión o cambio-Lista de especies en riesgo. Diario Oficial de la Federación. 2 a Sección, 6 de marzo de 2002.

Silva-Pérez M.L. 1998. Los bosques de coníferas del sur de la Cuenca de México: Fitosociología, diversidad y uso tradicional. Tesis de Licenciatura (Biología), Facultad de Ciencias,
Universidad Autónoma del Estado de México, Toluca, 82 pp.

Tejero-Díez D.J. y Arreguín-Sánchez M.L. 2004. Lista con anotaciones de los pteridófitos del estado de México, México. Acta Botanica Mexicana 69:1-82.

Toledo V.M., Carabias J., Toledo C. y González-Pacheco C. 1993. La Producción Rural en México: Alternativas Ecológicas. Fundación Universo Veintiuno y Prensa de Ciencias, México, D.F.

Tolmatschew A.I. 1971. Über einige quantitative Wechselbeziehungen der Foren der Erde. Feddes Repertorium 82:343-356.

Turner B.L. y Nesom G.L. 1998. Biogeografía, diversidad y situación de peligro o amenaza de Asteraceae de México. En: Ramamoorthy T.P., Bye R., Lot A. y Fa J. Comp. Diversidad Biológica de México: Orígenes y Distribución, pp. 545-561, Instituto de Biología, Universidad Nacional Autónoma de México, México, D.F.

Vázquez-García J.A. 1993. Cloud forest archipelagos: Preservation of fragmented montane ecosystems in tropical America. En: Hamilton L.S., Juvik J.O. y Scatena F.N. Eds. Proceedings of the International Symposium on Tropical Montane Cloud Forest, pp. 203-216, East West Center, UNESCO (Int. Hydrol. Program., IITF), San Juan, Puerto Rico.

Vega-Aviña R. 1982. Manual de la flora de la estación experimental de enseñanza e investigación y servicios forestales Zoquiapan. Tesis de Maestría, Colegio de Postgraduados. Chapingo, Estado de México, 364 pp.

Velázquez A. y Cleef. A.M. 1993. The plant communities of the volcanoes "Tláloc" and "Pelado", Mexico. Phytocoenologia 22:145-191.

Vibrans H. 1998. Native maize field weed communities in southcentral Mexico. Weed Research 38:153-166.

Villaseñor J.L. y Espinosa-García F.J. 2004. The alien flowering plants of Mexico. Diversity and Distributions 10:113-123.

Fecha de recepción: 26 de mayo de 2006

Versión corregida: 26 de octubre de 2006

Aceptado: 27 de octubre de 2006 
Apéndice 1. Listado de especies recolectadas en el bosque de oyamel del Cerro Tláloc.

Familia Especie

\section{DIVISIÓN POLYPODIOPHYTA}

Aspleniaceae Asplenium castaneum Schltdl. et Cham. Asplenium fibrillosum Pringle et Davenp. ex Davenp. Asplenium monanthes L.

Athyriaceae Cystopteris fragilis (L.) Bernh.

Dryopteridaceae Polystichum speciosissimum (A.Braun ex Kunze) Copel.

Polypodiaceae $\quad$ Pleopeltis polylepis (Roem. ex Kunze) T.Moore var. interjecta (Weath.) Mickel et Beitel Polypodium californicum Kaulf.

\section{DIVISIÓN PINOPHYTA}

$\begin{array}{ll}\text { Pinaceae } & \text { Abies religiosa (Kunth) Schltdl. et Cham. } \\ & \text { Pinus ayacahuite C.Ehrenb. ex Schltdl. } \\ & \text { Pinus hartwegii Lindl. } \\ & \text { Pinus montezumae Lamb. } \\ & \text { Pinus patula Schltdl. et Cham. } \\ & \text { Cupressus lusitanica Mill. }\end{array}$

\section{DIVISIÓN MAGNOLIOPHYTA}

CLASE LILIOPSIDA

Commelinaceae

Orchidaceae

Poaceae
Tradescantia crassifolia var. acaulis M.Martens et Galeotti Corallorrhiza macrantha Schltr.

Corallorrhiza striata Lindl.

Funkiella hyemalis (A.Rich. et Galeotti) Schltr.

Brachypodium mexicanum (Roem. et Schult.) Link

Cinna poiformis (Kunth) Scribn. et Merr.

Festuca amplissima Rupr.

Festuca orizabensis E.B.Alexeev

Festuca tolucensis Kunth

Festuca willdenowiana Schult. et Schult.f.

Muhlenbergia cf. tenuifolia (Kunth) Kunth

Muhlenbergia nigra Hitchc.

Muhlenbergia quadridentata (Kunth) Trin.

Poa annua L.

Stipa ichu (Ruiz et Pav.) Kunth

Trisetum virletii E.Fourn.

Vulpia myuros (L.) C.C.Gmel.

\section{CLASE MAGNOLIOPSIDA}

Asteraceae

Achillea millefolium L.

Archibaccharis hieracioides (S.F.Blake) S.F.Blake

Archibaccharis serratifolia (Kunth) S.F.Blake

Baccharis conferta Kunth

Baccharis multiflora Kunth

Baccharis serraefolia DC.

Bidens cf. triplinervia Kunth

Cirsium ehrenbergii Sch.Bip.

Conyza filaginoides (DC.) Hieron.

Chaptalia lyrata (Willd.) Spreng.

Erigeron galeottii (A.Gray) Greene

Eupatorium glabratum Kunth 


\begin{tabular}{|c|c|}
\hline Familia & Especie \\
\hline & $\begin{array}{l}\text { Eupatorium pazcuarense Kunth } \\
\text { Eupatorium prunellifolium Kunth } \\
\text { Eupatorium rhomboideum Kunth } \\
\text { Gnaphalium americanum Mill. } \\
\text { Gnaphalium attenuatum DC. } \\
\text { Gnaphalium chartaceum Greenm. } \\
\text { Haplopappus stoloniferus DC. } \\
\text { Parthenium bipinnatifidum (Ortega) Rollins } \\
\text { Pinaropappus roseus (Less.) Less. } \\
\text { Selloa plantaginea Kunth } \\
\text { Senecio angulifolius DC. } \\
\text { Senecio barba-johannis DC. } \\
\text { Senecio bellidifolius Kunth } \\
\text { Senecio callosus Sch.Bip. } \\
\text { Senecio cinerarioides Kunth } \\
\text { Senecio platanifolius Benth. } \\
\text { Senecio sinuatus Gilib. } \\
\text { Senecio toluccanus DC. } \\
\text { Sigesbeckia jorullensis Kunth } \\
\text { Sonchus oleraceus L. } \\
\text { Stevia jorullensis Kunth } \\
\text { Stevia monardifolia Kunth } \\
\text { Stevia salicifolia Cav. } \\
\text { Taraxacum officinale F.H.Wigg. } \\
\text { Verbesina oncophora B.L.Rob. et Seaton }\end{array}$ \\
\hline Berberidaceae & Berberis moranensis Schult. et Schult.f. \\
\hline Betulaceae & Alnus acuminata Kunth \\
\hline Boraginaceae & Lithospermum distichum Ortega \\
\hline Caprifoliaceae & $\begin{array}{l}\text { Symphoricarpos microphyllus Kunth } \\
\text { Lonicera pilosa (Kunth) Spreng. }\end{array}$ \\
\hline Caryophyllaceae & $\begin{array}{l}\text { Arenaria lanuginosa (Michx.) Rohrb. } \\
\text { Arenaria reptans Hemsl. } \\
\text { Stellaria cuspidata Willd. ex Schltdl. }\end{array}$ \\
\hline Cruciferae & Descurainia impatiens (Cham. et Schl.) O.E.Schulz \\
\hline Ericaceae & $\begin{array}{l}\text { Arbutus xalapensis Kunth } \\
\text { Comarostaphylis discolor (Hook.) Diggs } \\
\text { Pernettya prostrata (Cav.) DC. }\end{array}$ \\
\hline Euphorbiaceae & Euphorbia furcillata Kunth \\
\hline Fagaceae & Quercus laurina Bonpl. \\
\hline Gentianiaceae & $\begin{array}{l}\text { Gentiana spathacea Kunth } \\
\text { Gentianella amarella (L.) Börner } \\
\text { Halenia brevicornis (Kunth) G.Don }\end{array}$ \\
\hline Geraniaceae & $\begin{array}{l}\text { Erodium cicutarium (L.) L'Hér. ex Aiton } \\
\text { Geranium latum Small } \\
\text { Geranium lilacinum R.Knuth } \\
\text { Geranium potentillifolium DC. } \\
\text { Geranium seemannii Peyr. }\end{array}$ \\
\hline Grossulariaceae & Ribes ciliatum Humb. et Bonpl. ex Roem. et Schult. \\
\hline Hydrophyllaceae & Phacelia platycarpa (Cav.) Spreng. \\
\hline Lamiaceae & $\begin{array}{l}\text { Prunella vulgaris } \mathrm{L} . \\
\text { Salvia fulgens Cav. } \\
\text { Salvia gesneriflora Lindl. } \\
\text { Stachys coccinea Ortega }\end{array}$ \\
\hline Fabaceae & $\begin{array}{l}\text { Astragalus mollissimus Torr. } \\
\text { Lupinus campestris Cham. et Schltdl. }\end{array}$ \\
\hline
\end{tabular}




\begin{tabular}{|c|c|}
\hline Familia & Especie \\
\hline & Lupinus montanus Kunth \\
\hline Loganiaceae & Buddleia parviflora Kunth \\
\hline \multirow[t]{2}{*}{ Loranthaceae } & Arceuthobium abietis-religiosae Heil \\
\hline & Arceuthobium globosum Hawksw. et Wiens \\
\hline \multirow[t]{5}{*}{ Onagraceae } & Epilobium ciliatum Raf. \\
\hline & Fuchsia microphylla Kunth \\
\hline & Fuchsia thymifolia Kunth \\
\hline & Lopezia racemosa Cav. \\
\hline & Oenothera purpusii Munz \\
\hline \multirow[t]{2}{*}{ Oxalidaceae } & Oxalis alpina (Rose) R.Knuth \\
\hline & Oxalis corniculata L. \\
\hline Plantaginaceae & Plantago australis Lam. ssp. hirtella Kunth \\
\hline Polemoniaceae & Polemonium mexicanum Cerv. ex Lag. \\
\hline Polygonaceae & Polygonum aviculare L. \\
\hline Portulacaceae & Claytonia perfoliata Donn ex Willd. \\
\hline \multirow[t]{3}{*}{ Pyrolaceae } & Monotropa hypopitys L. \\
\hline & Monotropa uniflora L. \\
\hline & Orthilia secunda (L.) House \\
\hline Resedaceae & Reseda luteola L. \\
\hline \multirow[t]{4}{*}{ Rosaceae } & Acaena elongata L. \\
\hline & Alchemilla procumbens Rose \\
\hline & Fragaria mexicana Schltdl. \\
\hline & Potentilla staminea Rydb. \\
\hline \multirow[t]{2}{*}{ Rubiaceae } & Didymaea alsinoides (Cham. et Schltdl.) Standl. \\
\hline & Galium aschenbornii Nees et S.Schauer \\
\hline Salicaceae & Salix paradoxa Kunth \\
\hline Saxifragaceae & Heuchera orizabensis Hemsl. \\
\hline \multirow[t]{7}{*}{ Scrophulariaceae } & Castilleja tenuiflora Benth. \\
\hline & Mimulus glabratus Kunth \\
\hline & Penstemon campanulatus (Cav.) Willd. \\
\hline & Penstemon gentianoides (Kunth) Poir. \\
\hline & Sibthorpia repens (L.) Kuntze \\
\hline & Veronica persica Poir. \\
\hline & Veronica serpyllifolia var. humifusa (Dicks.) Vahl \\
\hline \multirow[t]{2}{*}{ Solanaceae } & Solanum cf. cardiophyllum Lindl. \\
\hline & Solanum stoloniferum Schltdl. et Bouché \\
\hline \multirow[t]{2}{*}{ Umbelliferae } & Eryngium monocephalum Cav. \\
\hline & Eryngium proteiflorum Delaroche \\
\hline Urticaceae & Parietaria pensylvanica Muhl. ex Willd. \\
\hline Valerianaceae & Valeriana clematitis Kunth \\
\hline
\end{tabular}

